\title{
Steigende Erfolgschancen
}

Fragestellung: Ist eine kognitive Verhaltenstherapie als Augmentation $\mathrm{zu}$ „treatment as usual“ (inklusive Pharmakotherapie) bei ambulanten Patienten mit einer medikamentös therapieresistenten Depression wirksam?

Hintergrund: Nur etwa $30 \%$ aller Patienten mit einer Depression sprechen vollständig auf die initiale medikamentöse Therapie an. Die Empfehlungen für weiterführende therapeutische Schritte haben bislang eine unzureichende Evidenz. Insbesondere wurde die Wirksamkeit der kognitiven Verhaltenstherapie (KVT) in derartigen Kollektiven bislang nicht in größeren randomisierten, kontrollierten Studien untersucht.

Patienten und Methodik: In diese randomisierte, kontrollierte, nationale Multicenterstudie wurden 432 Patienten mit einer depressiven Störung nach ICD-10 aus 76 Allgemeinarztpraxen in drei Städten Großbritanniens eingeschlossen. Voraussetzung war, dass die Patienten über mindestens sechs Wochen medikamentös antidepressiv behandelt wurden, ohne ausreichend auf die Behandlung ange-

Wiles $\mathrm{N}$, Thomas $\mathrm{L}$, Abel A et al. Cognitive behavioural therapy as an adjunct to pharmacotherapy for primary care based patients with treatment resistant depression: results of the CoBalT randomised controlled trial. Lancet 2013; 381: 375-84 sprochen $\mathrm{zu}$ haben (BDI > 14). Ausgeschlossen wurden Patienten mit bipolarer Störung, Suchterkrankungen, psychotischen Symptomen oder einer Psychotherapie innerhalb der letzten drei Jahre vor Studieneinschluss. Die Patienten wurden auf zwei Studienarme stratifiziert randomisiert. Während die antidepressive Behandlung durch den Allgemeinarzt, inklusive medikamentöser Veränderungen und psychiatrischer Konsultationen, in beiden Studienarmen nicht eingeschränkt wurde, erhielten die Patienten im Behandlungsarm der Studie zusätzlich 12 bis 18 Sitzungen einer KVT. Primärer Endpunkt der Studie war das Ansprechen auf die Behandlung, definiert als Reduktion des BDI-II um mindestens 50\% nach sechs Monaten. Patienten im Kontrollarm der Studie erhielten keine entsprechende Behandlung.

Ergebnisse: Durch die stratifizierte Randomisierung zeigten sich kaum Unterschiede zwischen den Patienten in beiden Studienarmen. Mehr als $80 \%$ der Patienten waren bereits länger als sechs Monate mittelschwer oder schwer erkrankt, hatten bereits früher depressive Episoden erlitten und waren medikamentös vorbehandelt. Während im Behandlungsarm nach sechs Monaten $46 \%$ der Patienten ein Ansprechen auf die Therapie zeigten, waren es im Kontrollarm lediglich $22 \%$ (Odds Ratio 3,26, p < $0,001$ bzw. Number needed to treat $=4)$. Ebenso reduzierten sich Angst und Panik in der KVT-Gruppe signifikant stärker und die Lebensqualität war höher. In der Nachuntersuchung nach zwölf Monaten bestätigten sich diese Ergebnisse. Die Rate der Studienabbrecher lag in beiden Armen bei etwa 16\%.

Schlussfolgerungen: Bei ambulanten Patienten mit depressiver Störung und unzureichendem Ansprechen auf die medikamentöse Behandlung stellt eine zusätzliche Verhaltenstherapie eine wirksame Erweiterung der Therapie dar.

\section{-Kommentar von Philipp Spitzer, Erlangen}

\section{KVT ist eine wirksame Ergänzung}

Erstmals konnte hier die Wirksamkeit der KVT als Augmentation bei medikamentöser Therapieresistenz in einer großen, randomisierten, kontrollierten Multicenterstudie nachgewiesen werden. Kritisch anzumerken ist, dass die Kontrollgruppe keinerlei vergleichbare Intervention erhielt, sodass für eine Placebowirkung nicht kontrolliert werden konnte. Es bleibt daher festzuhalten, dass der beobachtete Effekt nicht allein auf die KVT zurückzuführen ist, sondern zu einem unklaren Anteil der vermehrten Zuwendung unter den Studienbedingungen geschuldet ist. Die Schlussfolgerung, dass eine KVT für jene $70 \%$ der Patienten, die nicht ausreichend auf die medikamentöse Therapie ansprechen, eine wirksame Ergänzung ist, bleibt dadurch unberührt. Lediglich die Frage, ob andere Psychotherapieverfahren oder andere Arten persönlicher Zuwendung ähnlich wirksam sind, bleibt unbeantwortet.

Positiv hervorzuheben ist, dass das Patientenkollektiv aufgrund der wenigen Ausschlusskriterien, der geforderten Therapieresistenz und der Rekrutierung in Allgemeinarztpraxen repräsentativ für ein „Problemkollektiv“ ist. Die Patienten waren über-

wiegend chronisch erkrankt, multipel vorbehandelt und mittelschwer bis schwer betroffen. Hält man sich nun die hohe Effektivität der KVT in diesem Kollektiv vor Augen, lässt dies den Ruf der Autoren nach einer besseren Verfügbarkeit von Psychotherapieangeboten nur allzu gerechtfertigt erscheinen. Wir dürfen sehr gespannt sein auf die Auswertung der im Rahmen dieser Studie gesammelten sozioökonomischen Daten.

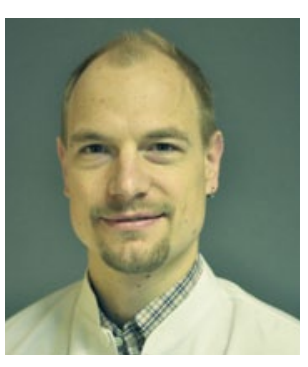

Dr. med. Philipp Spitzer, Erlangen

Psychiatrische und Psychotherapeutische Klinik Erlangen

E-Mail:Philipp.Spitzer@uk-erlangen.de 